\title{
Gradhiva
}

GRADHIV

Revue d'anthropologie et d'histoire des arts

$24 \mid 2016$

ARTchives

\section{Entretien avec Philippe Artières}

\section{Alice Aterianus-Owanga, Nora Greani et Philippe Artières}

\section{OpenEdition}

\section{Journals}

Édition électronique

URL : http://journals.openedition.org/gradhiva/3294

DOI : $10.4000 /$ gradhiva.3294

ISSN : 1760-849X

\section{Éditeur}

Musée du quai Branly Jacques Chirac

\section{Édition imprimée}

Date de publication : 7 décembre 2016

Pagination : 190-205

ISBN : 978-2-35744-094-4

ISSN : 0764-8928

Référence électronique

Alice Aterianus-Owanga, Nora Greani et Philippe Artières, «Entretien avec Philippe Artières », Gradhiva [En ligne], 24 | 2016, mis en ligne le 07 décembre 2019, consulté le 01 mai 2019. URL : http:// journals.openedition.org/gradhiva/3294; DOI : 10.4000/gradhiva.3294 



\section{Entretien avec Philippe Artières}

par Alice Aterianus-Owanga et Nora Greani

Historien spécialiste des écritures ordinaires contemporaines, ancien pensionnaire de l'Académie de France à Rome et actuellement directeur de recherche au CNRS, Philippe Artières a contribué à renouveler l'approche traditionnelle des archives. Par ses diverses collaborations avec des artistes, il offre un regard original sur la rencontre entre art et archives. 
1. L'Association pour l'autobiographie et le patrimoine autobiographique (APA) a été créée en 1992 dans l'objectif de collecter, conserver et valoriser des textes autobiographiques inédits.

2. Précisons que Daniel Fabre n'a pas déclaré l'importance d'archiver des autobiographies, mais il a reconnu l'importance du phénomène culturel de collecte et de conservation de l'écriture de soi, et a encouragé la comparaison de ses diverses déclinaisons en Europe. Voir Daniel Fabre, "Vivre, écrire, archiver", Sociétés \& représentations 13, 2002: 17-42.

3. Charles Double, État psychologique et mental d'un inverti parricide, 1905 , suivi de Impressions d'un condamné; Hermaphrodite mental!, Philippe Artières (éd.), Lille, Question de genre/GKC, 1995.
Alice Aterianus-Owanga et Nora Greani - Dans votre parcours de chercheur, vous vous êtes intéressé à des objets et des formes d'écriture qui s'écartent du discours historique classique et avez fréquemment collaboré avec des artistes autour d'archives de différentes natures. Cette approche nous a semblé faire écho à l'attention portée dans ce dossier aux usages des archives dans l'art extra-occidental et l'art ordinaire.

Philippe Artières - Concernant cet aspect-là, je voudrais préciser que je ne me situe pas du tout dans un travail réflexif, mais plutôt dans une pratique avec des artistes contemporains. J'envisage cette question du lien entre art et archives plus comme un récit d'expériences que quelque chose qui serait théorisable. Notre échange doit donc être entendu comme un témoignage.

A.A. et N.G. - Pour commencer, il serait intéressant de revenir sur la plasticité de la notion d'archive. Vous avez œuvré à élargir l'idée de l'Archive avec un grand $A$, notamment au travers de vos travaux sur les écrits de criminels. Quelle distinction faitesvous entre archives mineures et majeures?

Ph. A. - Cette nécessité de nommer des archives mineures, et du même coup de focaliser l'attention sur elles, est liée à un moment double dans I'histoire des archives. D'une part, au début des années 1990, on voit surgir les revendications de praticiens d'écritures personnelles à un moment où se développe la recherche sur les écrits personnels en Europe, notamment au travers de l'«Association pour l'autobiographie ${ }^{\mathbf{1}}$ », à l'initiative de Philippe Lejeune et de quelques-uns, dont j'étais. II était alors nécessaire d'insister sur l'importance d'archiver des écritures méconnues et dévalorisées. Ce mouvement était plutôt porté par des littéraires, bien qu'assez vite soutenu également par les sciences humaines - je pense à Michelle Perrot et, évidemment, à Daniel Fabre $^{2}$. D'autre part, on assiste à une valorisation des archives privées au sein de nouveaux lieux, dont l'Imec [Institut mémoires de l'édition contemporaine, à Caen] par exemple, où se constitue un panthéon éditorial, littéraire et des sciences sociales des intellectuels du $x x^{e}$ siècle.

Entre les deux, certaines archives semblaient ne pas être prises en compte alors qu'elles participaient de ces deux corpus. C'était tout ce qui était au fond de la boîte, à proximité du gisement, pourrait-on dire, des grandes figures.

Je pense à deux cas. À Lyon d'abord, en 1992, où j'ai travaillé sur un ensemble d'autobiographies de criminels de la fin du XIX ${ }^{e}$ siècle. Celles-ci correspondaient à une «sous-sous-cote», presque invisible, dans les archives du professeur Alexandre Lacassagne - père de l'anthropologie criminelle française - considérées comme un fonds annexe. J'ai alors essayé de valoriser ces archives mineures, au sein du fonds ancien de la bibliothèque municipale de Lyon, par des expositions ou encore par l'édition du récit autobiographique de Charles Double ${ }^{3}$. C'était une manière de valoriser ces individus restés dans l'ombre des savants de la Belle Époque; leurs 
archives mineures étant comme collées ou attachées aux archives personnelles d'hommes illustres.

À la même période, à l'invitation de Michelle Perrot, j'ai commencé à m'occuper du Centre Michel-Foucault. Quand nous avons entrepris de constituer un fonds d'archives autour du Groupe d'information sur les prisons [GIP4] fondé en 1971, beaucoup de chercheurs, à commencer par ses biographes, disaient: «Oui, bon, ce sont là les engagements de Michel Foucault, voilà... » Or il ne s'agissait pas à proprement parler des archives de Michel Foucault, mais d'un ensemble d'archives mineures en leur sein, attentif au «marmonnement du monde $\mathbf{5}$ ». De la même manière que les archives mineures de Lacassagne participaient du projet anthropologique qu'il cherchait à développer, celui d'une anthropologie de l'infime.

Si Foucault, avec Daniel Defert et d'autres, ne visait pas à constituer des archives, une sédimentation s'est cependant opérée autour de lui, et ce résidu n'intéressait alors personne. Ce qui est frappant, c'est que Foucault n'en était en réalité que le réceptacle. Les gens écrivaient au GIP, lui envoyaient des textes, des autobiographies, des récits de situation, parce qu'ils savaient que ce serait conservé quelque part, que ça se trouverait chez Foucault. C'était cela même qui m'intéressait chez ces deux chercheurs [Foucault et Lacassagne]. Ces archives témoignaient vraiment d'un regard spécifique et d'une invention, au sens d'une découverte. Lacassagne invente finalement l'informateur. II transforme le sujet observé en informateur. Et cette attention est absolument géniale dans le moment qui est le sien, en 1890, à une période où le savoir positiviste est dominant. De même, Foucault est l'un des premiers à prendre au sérieux la parole énoncée par les prisonniers et à l'entendre comme un discours politique légitime.

A.A. et N.G. - Vous évoquez les archives que Foucault a accumulées dans le cadre du GIP. Vous avez également fait un appel public à propos de Sida Mémoires et des archives personnelles des malades. Comment est née cette démarche?

Ph. A. - J'appartiens à la génération née en 1968, pour qui l'un des événements majeurs est la pandémie du VIH. En France, il s'agit du seul événement historique un peu équivalent à une guerre dont j'ai été témoin je mets de côté les récents attentats terroristes. J'ai travaillé au ministère de la Santé pendant cinq ans et, au sortir de cette expérience, je me suis dit: la totalité des archives personnelles, des écrits des personnes vivant avec le virus, ne sera pas conservée. J'ai expliqué à Olivier Corpet et à Albert Dichy $^{6}$ notre volonté de créer une association qui s'appellerait Sida Mémoires, capable de regrouper les archives autobiographiques des malades. C'était très important pour nous, à la fois en tant que chercheurs et témoins - je pense à Gilles Cugnon et à Catherine Viollet malheureusement morts depuis -, qui avions à cœur cette dimension militante. On se situait dans une double perspective mémorielle et scientifique. Il était symboliquement essentiel que ces archives collectées, écrits personnels de malades ordinaires, soient conservées auprès de celles des figures célèbres de l'écriture du sida, comme Hervé Guibert ou Bernard-Marie Koltès. Ainsi, me disais-je,
4. Lancé par Michel Foucault, Jean-Marie Domenach et Pierre Vidal-Naquet, le GIP (1971 1972) avait pour objectif d'alerter l'opinion publique sur les conditions de détention dans les prisons françaises et de libérer la parole des détenus.

5. Michel Foucault, Histoire de la folie à l'âge classique: folie et déraison, préface, Paris, Plon, 1961, p. VII.

6. Respectivement l'un des fondateurs et le directeur littéraire de l'Imec. 


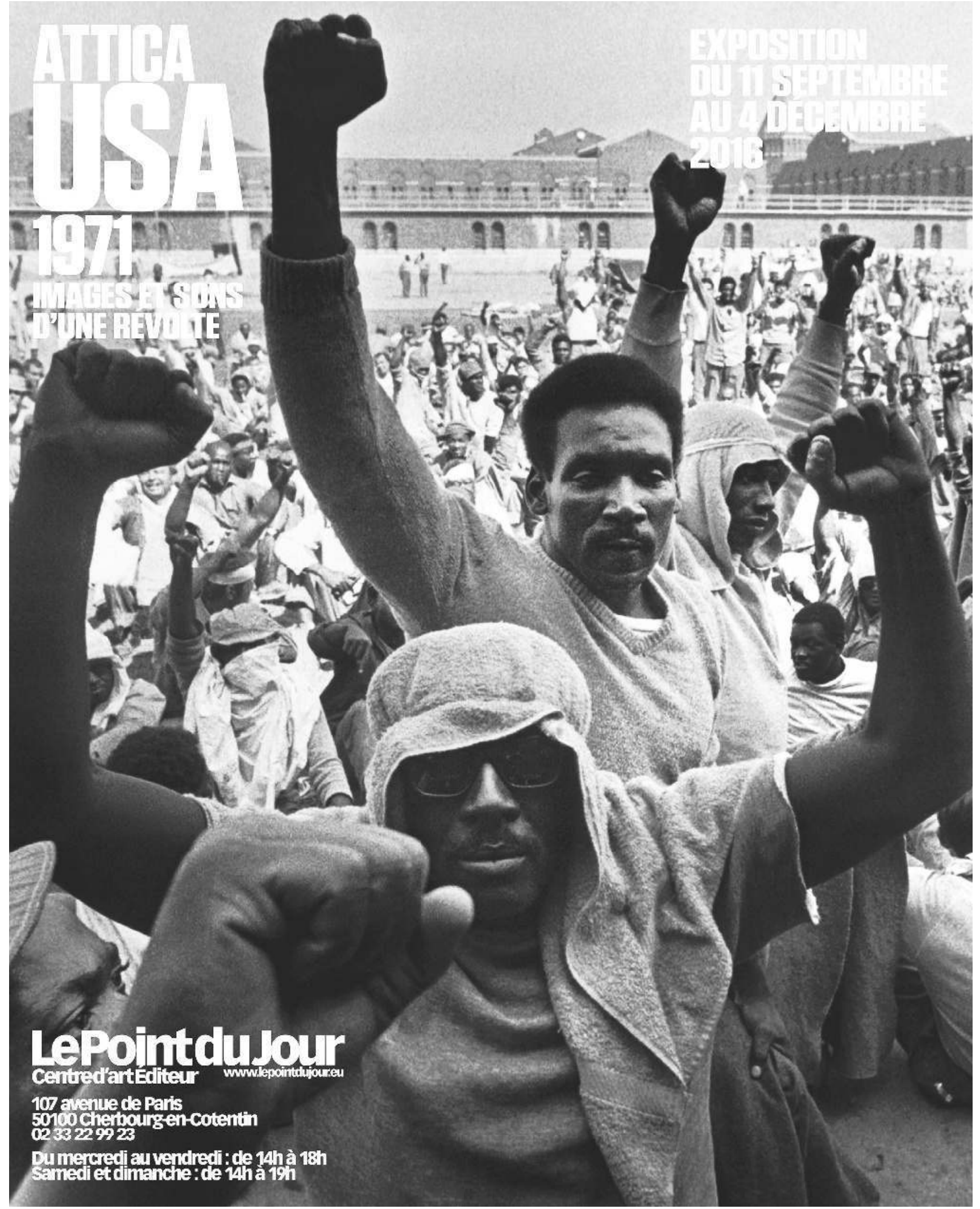


les gens venus voir des archives d'hommes illustres tomberaient sur celles des «infâmes». II ne s'agissait pas de considérer ces écritures comme étant littéraires, mais d'introduire des écrits ordinaires en ce lieu de distinction qu'est I'Imec (celui des archives littéraires et éditoriales) et de permettre ainsi une histoire sociale de l'écriture autour d'un fait tel que la pandémie du VIH.

Nous lançons donc un appel en 1999, soit trois ans après l'arrivée des antiprotéases et des premières trithérapies. L'hécatombe continue. On est juste après le pacs: la question de la transmission des biens est en cours de résolution. II convient de rappeler qu'auparavant, après la mort d'une personne, la famille débarquait et demandait au conjoint survivant: «Vous êtes qui, Monsieur? Comment ça, mon fils était homosexuel? » Leur nonexistence juridique mettait les survivants à la rue et les papiers du défunt, la plupart du temps, à la poubelle. Très souvent, des amis des personnes malades avaient encore des documents, probablement les seules traces qui restaient. Nous avons ainsi voulu éviter une collecte agressive en invitant plutôt à la constitution d'archives collectives, où les copies voisineraient avec les documents originaux. C'était là l'intensité de cette quête pour Sida Mémoires: retrouver la trace avant qu'elle ne disparaisse, lutter contre ce qui relevait du désir social d'effacement d'un événement, y compris d'ailleurs pour les proches. Car la perte de l'autre est insupportable: à 30 ans, on préfère oublier la perte d'une personne aimée, on a envie de continuer à vivre, de ne pas être dans le deuil. Nous avons donc voulu respecter absolument cette tension. II en est résulté une certaine opposition entre la mémoire et la recherche.

A.A. et N.G. - Dans votre travail de collecte des mémoires, vous semblez aller au-delà de certaines frontières disciplinaires. Vous paraît-il justifié de comparer votre démarche à celle d'un ethnographe, notamment dans votre recherche autour de Sida Mémoires?

Ph. A. - Si on entend par là le fait de documenter le passage entre la collecte des documents et leur inventaire, en un certain sens oui. Mais je ne me sens pas du tout ethnographe. Je suis très historien, attaché à l'idée qu'il n'y a d'histoire qu'à partir du moment où des archives sont partagées. On ne peut écrire une histoire du sida qu'à condition qu'existent des archives consultables par tout le monde. On ne peut écrire une histoire des mutineries dans les prisons seulement si plusieurs chercheurs ont accès aux archives. L'idée d'un terrain propre à tels ou tels chercheurs n'existe pas. Ce qui m'importe depuis toujours, c'est moins d'être un historien ou un ethnographe que d'être un passeur, celui qui aide à traverser le fleuve de la mort. C'est d'une ambition folle, je le reconnais volontiers.

Ma mission, si l'on veut, de chercheur au CNRS - il n'y a là rien de mystique - est très inscrite... J'ai les pieds dans la terre, voire dans le trou, dans la fosse. Dans nos sociétés, on donne à l'historien ce pouvoir-là, celui de transformer, selon une croyance qui remonte à Jules Michelet et sa conviction de ressusciter des morts. J'y ai régulièrement recours pour répondre à des demandes sociales. Prenons l'exemple d'Attica, une mutinerie

\section{ci-contre}

fig. 1

Affiche de l'exposition

Attica, USA 1971. Images et sons d'une révolte, 11 septembre - 4 décembre 2016, Cherbourg, Le Point du Jour; avec la photographie de Bob Schutz, Prison d'Attica, 10 septembre 1971. 


\section{fig. 2}

Album d'Alexandre

Lacassagne lors de

l'exposition Tatoueurs

Tatoués, 2014-2015,

musée du quai Branly

(c) Manon Coine.

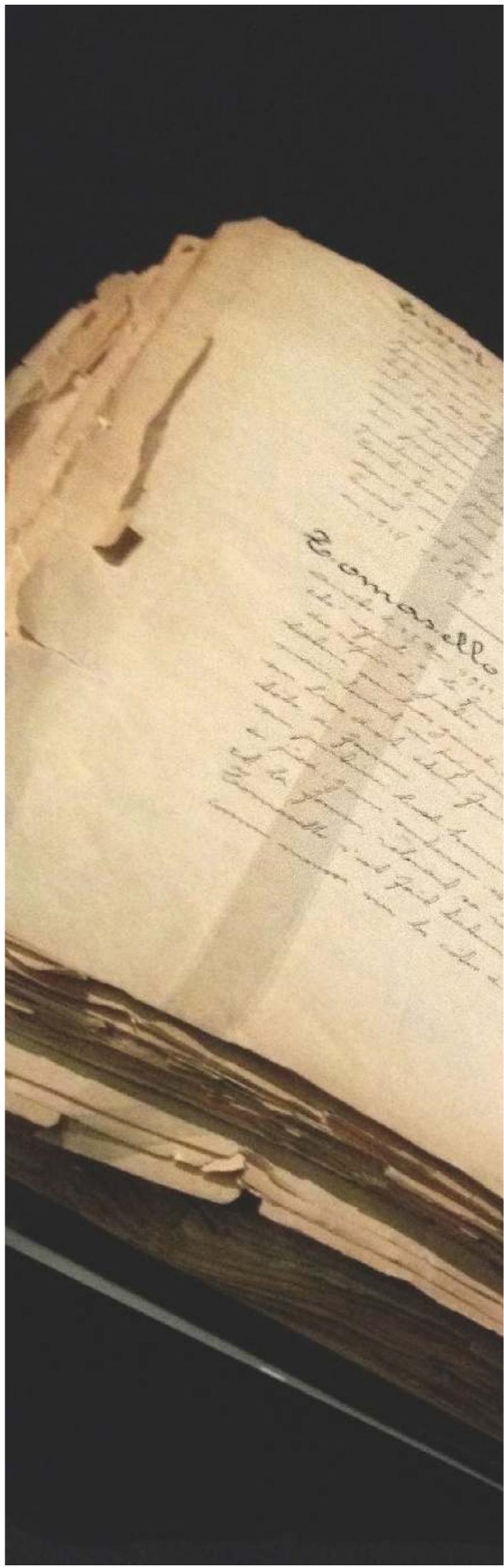




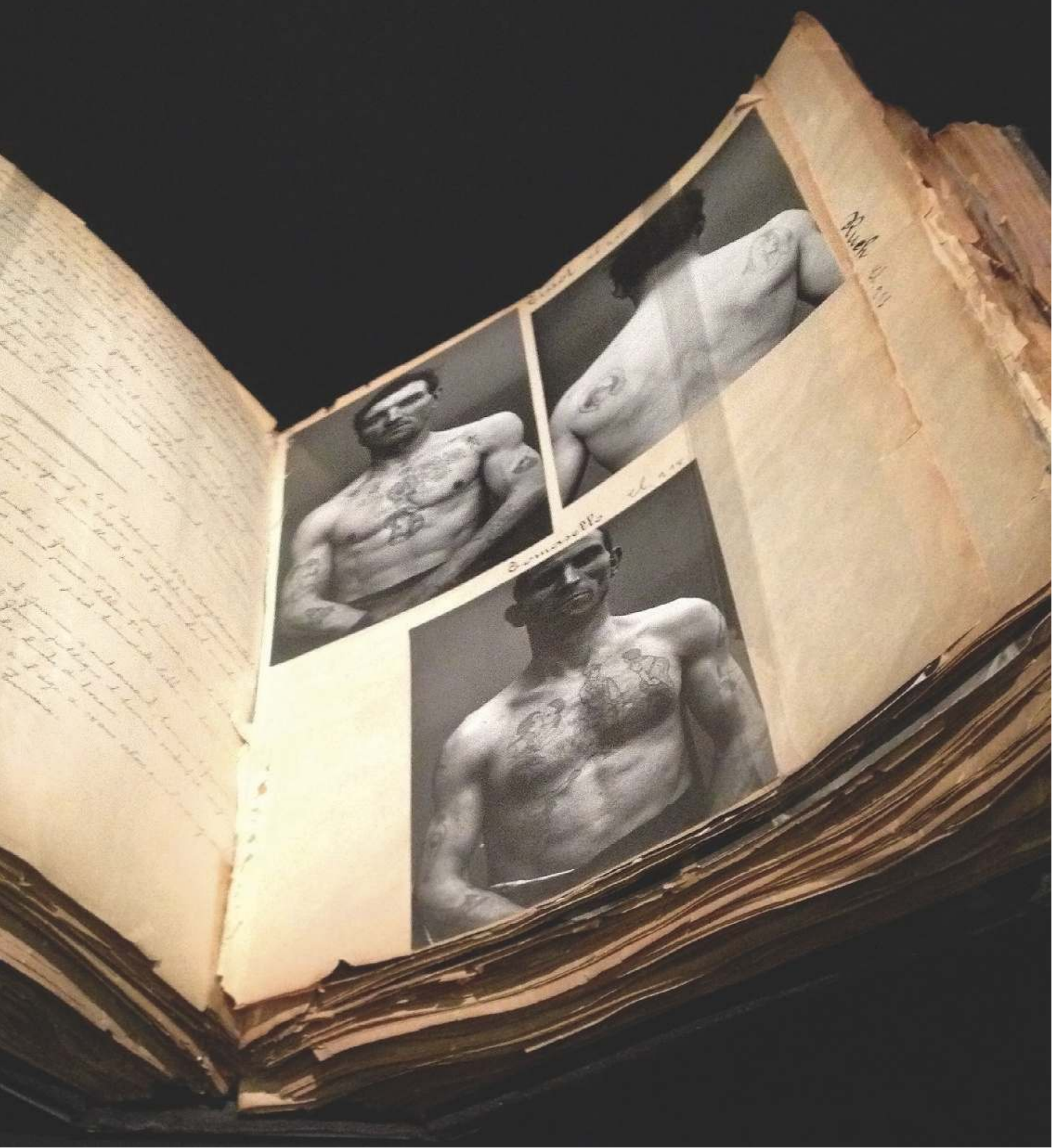




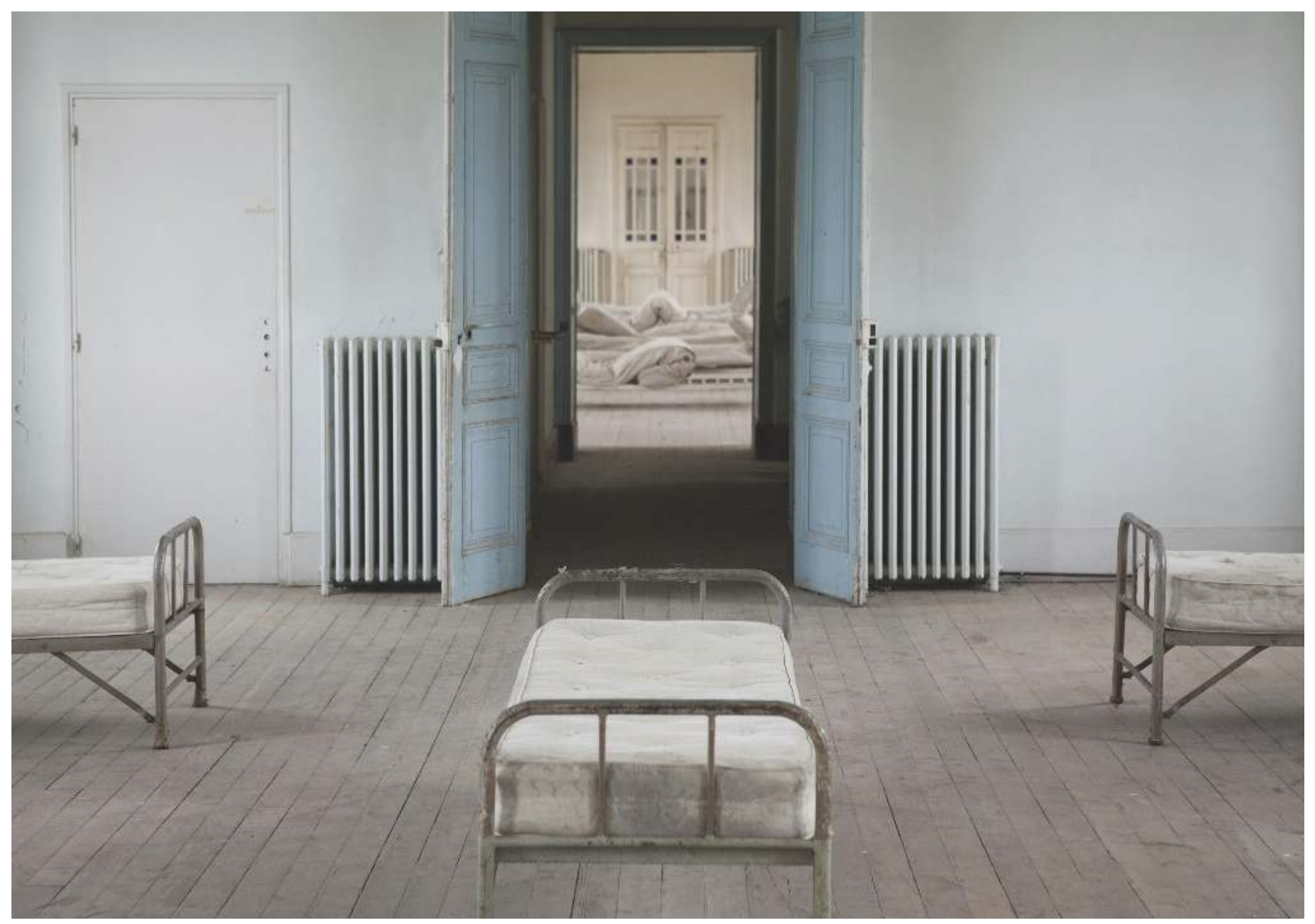


qui a eu lieu dans une prison de l'État de New York en 1971, alors que Rockefeller était gouverneur, et Nixon président. Ce soulèvement à la fois des Noirs et des Latinos se solda par quarante morts. Mais contrairement aux Black Panthers ou aux événements de la côte Ouest autour de George Jackson, ce fait majeur a été totalement évacué de l'histoire américaine, voilà pourquoi il m'intéresse depuis longtemps. Je prends alors contact avec l'avocate des «Attica Brothers » qui dispose d'énormément d'archives et me confie, en 2015, celles qui sont en sa possession - des photos de morts. Je sors donc des États-Unis avec ces photos. Mais l'avocate meurt brutalement et j'en deviens alors le gardien malgré moi. Depuis lors, mon projet d'en faire à la fois un livre et une exposition au sein d'un centre d'art, le Point du jour de Cherbourg, est lesté par le devoir de faire connaître ces archives. Il faudra d'ailleurs que l'exposition soit aussi montrée en Amérique du Nord, dans la grande ville la plus proche d'Attica [Toronto].

Ce travail fait suite à un projet précédent que j'ai mené avec le photographe Mathieu Pernot. Nous avions été invités par la Fondation Bon Sauveur de Picauville à travailler sur leurs archives psychiatriques. Réunis par l'éditeur Le Point du jour, nous n'avons pas été choisis au hasard. En effet, nous avions déjà un peu travaillé ensemble et partagions un rapport semblable aux archives ${ }^{7}$. À Picauville, nous avons découvert des archives mineures, communes à tous les asiles départementaux depuis 1837: toutes les archives de la vie dans l'institution, qui ne sont tout simplement ni valorisées ni même conservées. Ce qui était intéressant dans cet hôpital, c'était que la mémoire du lieu avait croisé celle de l'histoire mondiale: Picauville avait été en partie détruit lors du débarquement de juin 1944. S'imposait donc le besoin de faire récit malgré les silences.

\section{A.A. et N.G. - II vous est donc arrivé d'être à l'initiative de} la constitution de fonds d'archives, et d'autres fois, en revanche, vous en êtes devenu presque malgré vous le gardien?

Ph. A. - Oui, je dirais même un peu à mon corps défendant, parce que c'est lourd quand même. C'est ce que j'ai essayé d'aborder dans mon dernier livre qui tourne autour de la question suivante: que fait-on des morts et de leurs traces ${ }^{8}$ ? Pour tenter d'y répondre, nous avons, avec mon éditeur, tenté de faire du livre une forme, en l'occurrence un accrochage de récits. La tentative de production de savoir par l'accrochage m'apparaît efficace car la place y est laissée à celui qui lit et qui regarde, et c'est très important. Comment la littérature ou l'art contemporain réussissent-ils à faire entendre la voix oubliée des morts: voilà ce qui m'intéresse. Je pense à une pièce extraordinaire présentée à Paris au début de l'année 2016 à la galerie Marian Goodman, Ashes de Steve McQueen ${ }^{9}$. On y voit un jeune homme noir dans une barque, sur la mer des Caraïbes. Il est filmé, il est très beau, il est quasiment iconique! II descend, il plonge, il rit. De l'autre côté de l'écran, on voit un homme en train de fabriquer un tombeau en ciment. On en suit la construction de bout en bout, jusqu'à l'écriture de l'inscription sur la stèle. II s'agit du tombeau de ce jeune homme, un dealer qui est mort. C'est très simple, mais c'est un dispositif parfait au sens où il touche au but. Je me disais en voyant cette œuvre: «Ah! Si un jour j'arrivais à faire ça en tant
7. Durant trois ans, le photographe français Mathieu Pernot et Philippe Artières ont réalisé une recherche dans les archives de l'hôpital psychiatrique de Picauville en explorant tant les lieux que les documents conservés (dossiers médicaux, cartons, pochettes, classeurs de cartes postales, photos en noir et blanc, films, diapositives en couleurs). Cette recherche commune aboutit en 2013 à une exposition et à un livre: Philippe Artières et Mathieu Pernot, L'Asile des photographies, Cherbourg/Octeville, Le Point du jour.

8. Philippe Artières, Au fond, Paris, Seuil, 2016.

9. Voir à ce propos: http://mariangoodman. com/exhibition/2602/ press-release

\section{ci-contre}

fig. 3

Mathieu Pernot, Le Dortoir des agités, Picauville, 2010, installation sur le site de l'hôpital psychiatrique de Picauville (C) Mathieu Pernot, avec l'aimable autorisation de la galerie Éric Dupont. 
10. "Archives Duby, un inventaire provisoire", in Patrick Boucheron et Jacques Dalarun (dir.), Georges Duby, portrait de I'historien en ses archives, Paris, Gallimard, 2015: 26-57.

11. "Sophie Calle. M'as-tu vue", Centre Pompidou, Paris, 19 novembre 2003 15 mars 2004. qu'historien! », parvenir à produire par l'Histoire - et non par l'art - ce que cette pièce m'a fait à moi, physiquement. II se trouve que je connais assez bien la Caraïbe, et cette œuvre me semble véritablement inscrire un moment d'histoire violente. Ce n'est pas que cela m'émeut. Je ne pleure pas, je ne suis pas dans l'émotion. Mais soudain, ça surgit! Et c'est quand même ce que l'on attend d'un livre en sciences sociales, le refermer en se disant: "Oui, j'en ai vu émerger quelque chose.»

Regardons trente ans en arrière, si on prend le cas de Georges Duby (ou celui de Michel Leiris, évidemment, pour qui l'écriture était bien l'écriture littéraire): historien et médiéviste, Duby, tout en écrivant, ne cesse de chercher des formes, comme l'ont bien montré Patrick Boucheron et Jacques Dalarun ${ }^{10}$. Mais je pense qu'il y a eu un moment où l'histoire (la discipline) a fermé les écoutilles de la langue. De même que la sociologie et beaucoup d'autres disciplines... Comme si l'écriture était devenue sale et qu'il y avait un risque de devenir écrivain ou artiste.

\section{A.A. et N.G. - À propos de la représentation par les artistes d'une certaine vision de l'histoire, pourriez-vous nous en dire plus sur l'appropriation d'archives dans l'art?}

P. A. - Les archives - des vidéos familiales à celles du cabinet de De Gaulle par exemple, sur lesquelles je travaille en ce moment - ont une matérialité et une patrimonialisation très différentes. Comme vous le disiez précédemment, c'est un objet très plastique. Rappelons qu'elles sont régies et définies par la loi, celles de 1979 et de 2002. Leur usage par les artistes est donc extrêmement réglementé.

On a tendance à considérer les expositions d'archives ennuyeuses; celles-ci souffrent d'une représentation très négative: un long alignement de tombes devant lequel on passe sans que le regard ne soit capté. Cela tient bien sûr au fait que les archives ont longtemps été montrées comme des reliques qu'on ne pouvait que contempler, sans jamais les manipuler ni les plier. Leur horizontalité était comme entendue. Je crois que les artistes contemporains ont modifié cette situation en dépliant les liasses.

Je dis les artistes mais, en réalité, rares sont ceux qui travaillent dans les archives. Prenons le cas emblématique de Sophie Calle. Elle n'a jamais travaillé sur des archives mais à partir d'objets trouvés, tels que l'agenda, qui ne se trouvent pas «aux objets trouvés». Elle ne travaille pas sur un ensemble. Elle se saisit de photos qu'elle trouve, par exemple celles qu'elle avait exposées au Centre Pompidou ${ }^{11}$ de cette jeune femme disparue dont elle n'avait que quelques négatifs voilés, mis en scène et en espace... Mais ce n'est pas là, à mon sens, un usage de l'archive.

Rien à voir avec ces artistes qui constituent eux-mêmes des archives ou deviennent des collecteurs d'archives. Je pense notamment à ce qui s'est fait autour de la photographie au Moyen-Orient et à l'Atlas Group de Walid Raad. Cet artiste libanais a inventé une fiction de collecte collective 


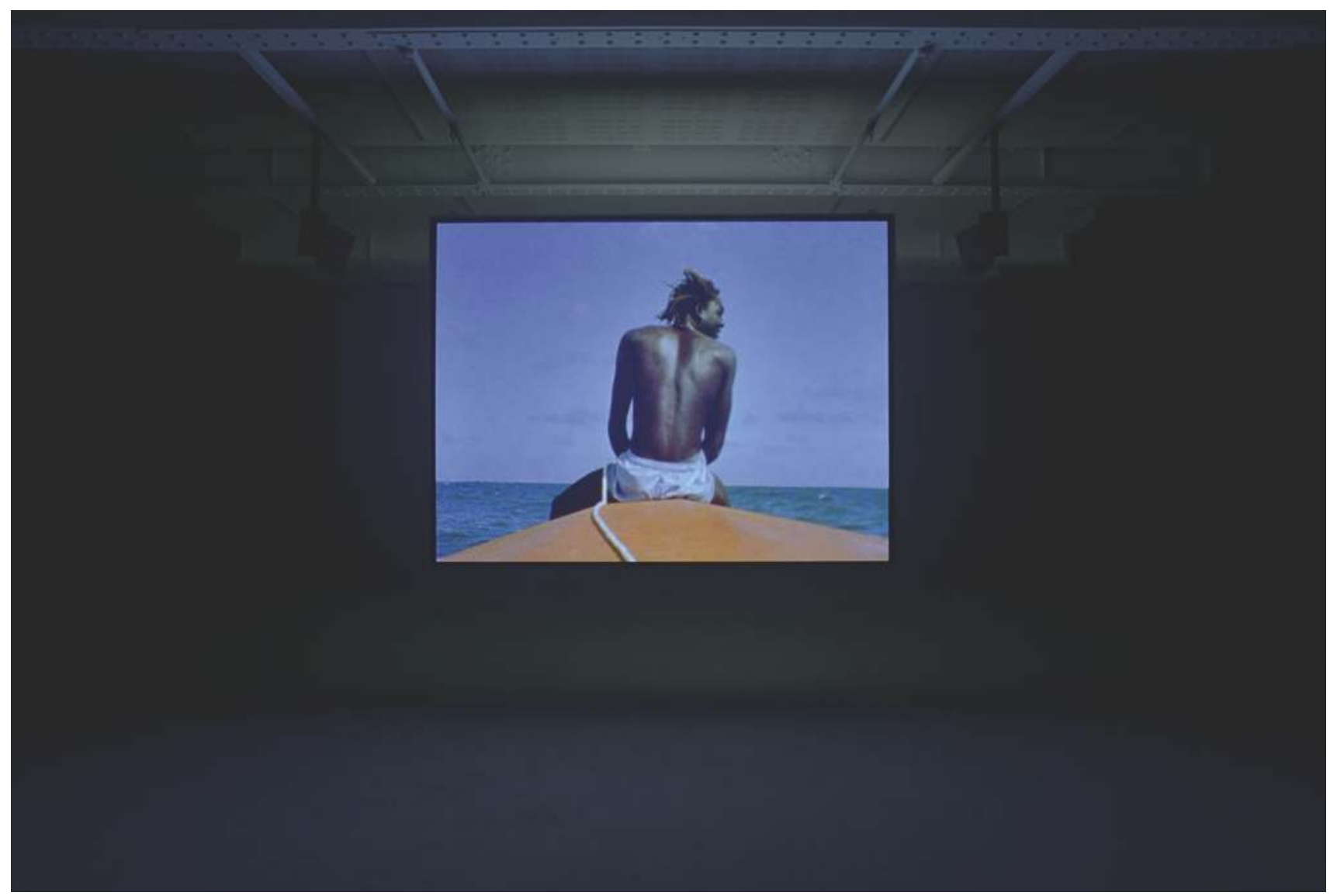

fig. 4

Steve McQueen, Ashes,

2002-2015, installation à la

Marian Goodman Gallery,

Paris, 2016. Avec l'aimable

autorisation de l'artiste et

de la Marian Goodman

Gallery. Photo Rebecca

Fanuele. 
12. L'Atlas Group est un projet de l'artiste Walid Raad qui s'attache à produire et conserver des documents sur l'histoire du Liban en inventant des archives et des documents par un détournement des notions d'histoire et d'archive. À ce propos, voir Stefanie Baumann, "Archiver ce qui aurait pu avoir lieu. Walid Raad et les archives de l'Atlas Group ", Conserveries mémorielles 6, 2009 [en ligne], disponible sur: http://cm.revues.org/381 (consulté le 18 juillet 2016).

13. Philippe Artières, Le Livre des vies coupables: autobiographies de criminels (1896-1909), Paris, Albin Michel, 2000.

14. Philippe Artières, "L'inscription dans un courant historiographique majeur ", Sociétés \& représentations 40, 2015 : 348

15. Annette Wieviorka, L'Ėre du témoin, Paris, Plon, 1998. d'archives historiques sur la guerre du Liban dont il est en réalité le seul auteur ${ }^{12}$.

II n'y a aucun jugement de ma part. Je ne dis pas que l'historien fait quelque chose de plus ou de mieux que l'artiste, de plus juste ou éthiquement acceptable. II ne s'agit pas du tout d'une question d'éthique, mais de production de savoir et d'intelligibilité... qui ne porte pas cependant sur un individu. II ne s'agit pas d'être fidèle, parce que de toute façon, on est dans l'infidélité et la trahison permanentes. Dans le premier texte que j'ai publié, au début du livre Les Vies coupables, je me souviens avoir écrit: “L'historien est un voleur ${ }^{13}$ ", comme l'archéologue qui rentre dans le tombeau et devient un pilleur. C'est fondamental car, quoi qu'il en soit, reste ce geste qui fait que l'on pénètre là où on n'a pas été invité. II est faux de dire que les archives sont le lieu des historiens. Elles sont celui de l'État, que les archives privées viennent compléter. Mais on y vient toujours par effraction et je refuse de dire qu'un historien serait alors plus légitime qu'un artiste ou un écrivain. Les artistes et les écrivains ont appréhendé bien avant les historiens cette matière très difficile à attraper qu'est la mémoire de l'événement comme participant de l'événement lui-même.

\section{A.A. et N.G. - Vous avez rendu compte ailleurs ${ }^{14}$ d'une forme d'inquiétude concernant la mise en concurrence actuelle du travail de l'historien avec celui de l'artiste. Pourriez-vous re- venir là-dessus?}

Ph. A. - J'ai vécu deux moments très différents où les savoirs en sciences sociales ont été particulièrement mis à mal. Tout d'abord celui qu'Annette Wieviorka appelait l'ère du témoin ${ }^{15}$, consistant à croire qu'il existerait une parole brute. Puis, l'époque où les artistes ont découvert les archives. Je pense notamment à tout ce courant postcolonial que j'ai pu observer lorsque j'étais au MAC VAL [musée d'Art contemporain du Val-de-Marne], au travers des pièces de Mathieu Abonnenc par exemple, ou encore au pavillon belge à la Biennale de Venise en 2015. On voit bien comment les artistes descendent sur les archives pour opérer une sorte de razzia, mis à part quelques-uns qui se disent qu'il y a peut-être quelque chose à inventer dans un dialogue avec les sciences sociales.

Jean-Christian Bourcart par exemple, un photographe singulier qui a à peu près tout fait - diverses commandes mais aussi une œuvre -, m'a proposé de me pencher sur ses archives et de les inventorier. J'aimerais que ce travail aboutisse à un lieu de consultation parce que c'est quelqu'un qui a photographié tout le monde aux États-Unis, en France! Vous voulez une photo de Pol Pot, de Carl Lewis, du président d'Act Up, et en même temps de Sarajevo ou du tremblement de terre de je ne sais où? Or Bourcart s'empare de toutes ses archives pour les brûler, les vendre, les "dealer", les donner.

C'est intéressant car cela reproblématise la question des archives et de leur marchandisation, qui est quand même le point ultime de leur artification: si les archives font leur entrée sur le marché de l'art, c'est bien le signe 
que leur artification a réussi. Cela concerne les archives d'artistes, mais aussi parfois celles de chercheurs en sciences sociales, comme Michel Foucault par exemple, dont la BNF [Bibliothèque nationale de France] a acquis les archives pour la somme de quatre millions d'euros. II y a bien là patrimonialisation, et une exposition aura lieu un jour, mais à quoi ressemblera-t-elle? Comment va-t-on montrer ses fiches? Au Centre Pompidou, Nathalie Léger et Marianne Alphand avaient réussi à présenter les archives de Roland Barthes de façon intéressante ${ }^{16}$. Ces deux commissaires avaient en effet choisi de donner à voir les fiches de travail de Barthes en les exposant toutes sur plusieurs mètres de hauteur, en empêchant certes parfois la lecture, mais en en faisant à la fois une carte et une stèle, un dépliement et un monument. De cette sorte de grand mur absolument illisible pour un chercheur, elles ont fait un objet contemporain, une œuvre d'art.

A.A. et N.G. - Suite à la publication du Goût de l'archive par Arlette Farge et à une série d'autres événements, les archives sont devenues l'archive: un objet singulier que l'on manipule, que l'on dépouille. D'objet-lieu, vous expliquez que les archives sont devenues corps. Pourriez-vous en dire plus sur ce devenircorps des archives?

Ph. A. - Dans Le Goût de l'archive, Arlette Farge propose l'idée que l'institution des archives est à la fois un lieu, un bâtiment, et ce qu'on y consulte. Au lieu de banaliser les archives, elle réifie la relique en en parlant au singulier. L'historien, lui seul, a le pouvoir de la toucher au prix d'un tas de petits rituels, de rites, d'épreuves: il faut rentrer, marcher, faire silence, et enfin on vous l'apporte...

Le Goût de l'archive est un livre très important quant au rapport d'une société à ses archives, dont Arlette Farge se fait le symptôme. Mais c'est très loin de nos pratiques actuelles, décrites notamment par Yann Potin ${ }^{17}$. Ce qui était sans doute valable à la bibliothèque de l'Arsenal ${ }^{\mathbf{1 8}}$ ne l'est plus pour un historien qui se rend aujourd'hui aux archives départementales ou à Pierrefitte ${ }^{19}$. II n'y a plus aucun fétichisme. Pour qui va dans les magasins des archives, c'est plutôt Amazon.com!

L'idée selon laquelle les archives seraient le lieu du secret persiste toujours. Mais au vu du nombre d'artistes qui y interviennent, on voit bien que ce sont des espaces ouverts. Par exemple, lorsque les Archives nationales ont accueilli Nicolas Frize pour une résidence, il y a fait à peu près ce qu'il voulait. II a notamment transformé des boîtes et des documents en instruments de musique; il a investi les magasins des réserves après de longues conversations avec tous les agents (des conservateurs aux magasiniers) et a inscrit certains de leurs propos sous forme de sentences qui ornaient les couloirs.

Cela ne signifie pas qu'il n'y ait ni éléments classifiés, ni abus de pouvoir. Mais si ceux-ci existent bien, ils correspondent à une critique de l'État, et non à celle des archivistes ou d'une institution.
16. "Roland Barthes", exposition au Centre Pompidou, Paris, 27 novembre $2002-$ 10 mars 2003.

17. Voir notamment Yann Potin, "L'historien en "ses" archives", in À quoi pensent les historiens? Faire de l'histoire au $x \times I^{e}$ siècle, Christophe Granger (dir.), Paris, Autrement, 2013: 101-117.

18. Lieu où se rend Arlette Farge.

19. Site des Archives nationales. 


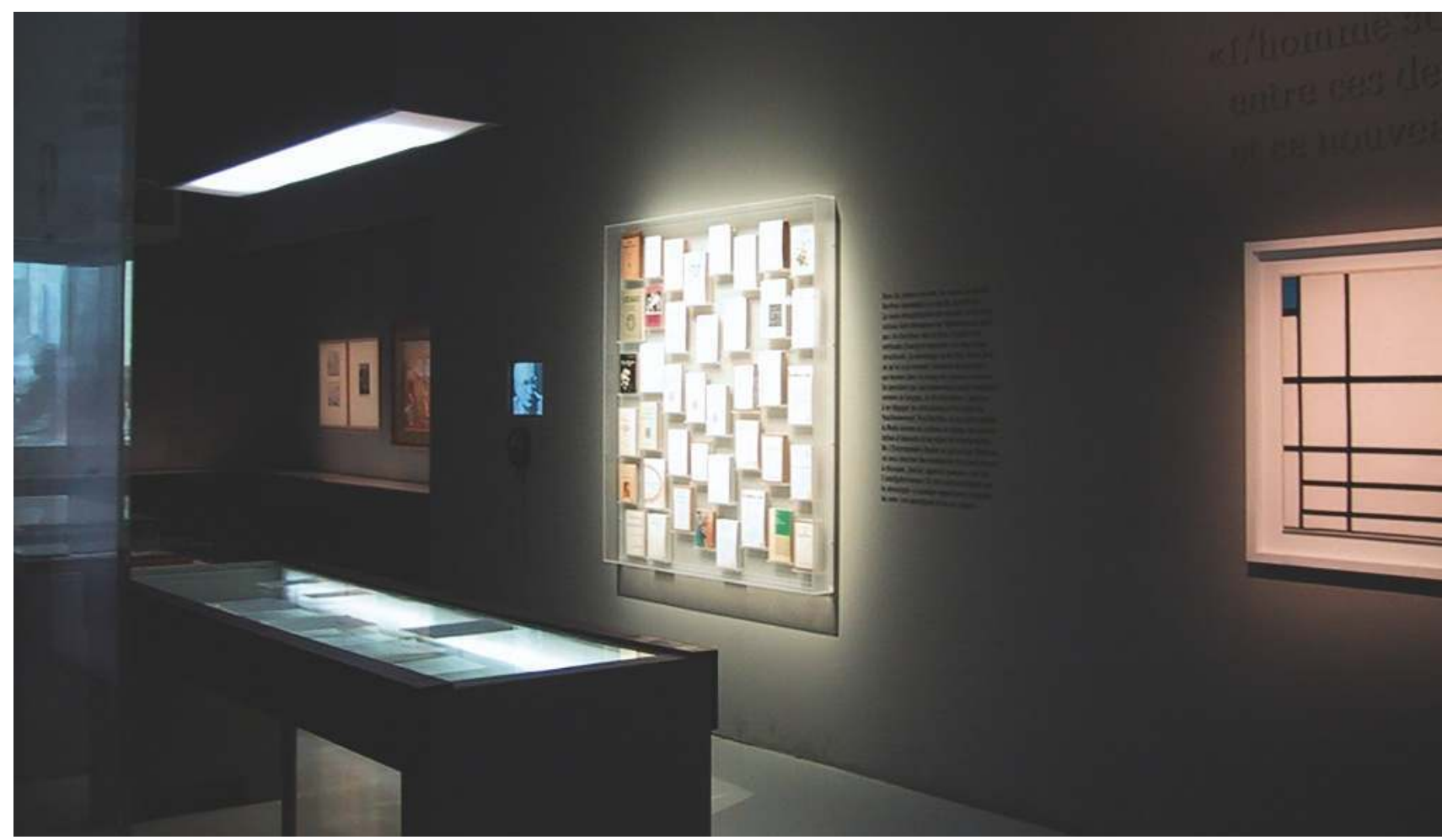


En ce moment, je travaille avec Emmanuelle Giry [conservateur aux Archives nationales] sur un projet d'exposition pour le cinquantenaire de $1968^{20}$. C'est très impressionnant d'ouvrir ces boîtes qui ne l'ont jamais été, puisque la loi sur les archives n'autorise leur consultation qu'au bout de cinquante ans. On découvre donc tout cela et c'est sidérant. Encore une fois, ce n'est pas le général de Gaulle qui m'intéresse, mais ce geste, cet acte d'archivage. Yves Cohen en parle très bien à propos des écrits de Staline ${ }^{21}$. Je ne compare pas du tout Charles de Gaulle à Staline, mais je me réfère à cette écriture appréhendée comme un acte performatif. Quand de Gaulle dit: “Licenciez les journalistes», ou quand il écrit: «ll faut reprendre l'Odéon", ça veut dire quoi? Ce document n'est pas la trace d'un acte, il est l'acte lui-même.

Dans ce projet d'exposition [sur le cinquantenaire de Mai 68], il y aura une salle de lecture avec des fac-similés. Peu importe qu'il s'agisse de photocopies, cela permettra néanmoins de voir une liasse et non un fragment équivalent à une sorte de relique ou d'ossement.

On ne peut pas dire qu'il n'y ait qu'un rapport mental au document, parce qu'il est là, on le tient. C'est peut-être aussi ce que je reproche au Goût de l'archive: ce singulier [«l'archive»] qui produit quelque chose de monstrueux. Ce n'est pas contre des institutions culturelles précises que je dis ça, mais quand vous regardez les inventaires des bibliothèques, à la BNF ou à l'Imec par exemple, c'est souvent du pièce à pièce. Or les archives sont un dossier. Ce n'est pas folioté de 1 à 30 aux Archives nationales, c'est plutôt de l'ordre du trente mètres linéaires!

En même temps, si l'on parle des archives du général de Gaulle, beaucoup de choses ont été jetées avant que ce soit archivé. Le producteur du fonds, surtout si c'est un ministre, a opéré un tri parmi les documents et a parfois pris avec lui certains dossiers. Très peu de choses sont aujourd'hui conservées par rapport à ce que l'institution a produit. Malgré ces manques, on retrouve cependant des séries. Travailler en essayant de reconstruire des séries d'événements d'écriture ou de réunir des ensembles d'archives m'intéresse beaucoup. L'idée de la pièce unique est très artificielle et participe d'une artification, y compris de la part des historiens: la belle pièce, le beau cas.

J'ai tendance à penser que l'archivage est une forme d'écriture. C'est-àdire qu'au fond l'archivage est non seulement une pratique de soi mais aussi une écriture de soi. Je pense évidemment à Foucault et à ce qu'il disait d'une esthétique de l'existence: c'est-à-dire construire sa vie comme une œuvre d'art, et en même temps son propre tombeau, son propre mausolée ou sa propre sépulture. Parce que, je l'ai déjà dit, les archives ont quand même beaucoup à voir avec la disparition. II est difficile de faire abstraction de ce rapport à la mort, difficile de ne pas penser les archives en omettant cette dimension si bien analysée par Michel de Certeau.
20. Voir Michelle Zancarini-Fournel et Philippe Artières (dir.), 68: une histoire collective (1962-1981), Paris, La Découverte, 2015.

21. Yves Cohen, Le Siècle des chefs: une histoire transnationale du commandement et de l'autorité (1890-1940), Paris, Éditions Amsterdam, 2013.

\section{ci-contre}

fig. 5

Vue de l'exposition RB /

Roland Barthes au Centre

Pompidou, 27 novembre

2002-10 mars 2003

Photo (c) agence nc, Paris. 